\title{
Cibermedios y agenda-setting: la configuración de la agenda mediática internacional
}

\author{
Javier OdRIOZOLA CHENÉ \\ Universitat Pompeu Fabra \\ javodriozola@hotmail.com
}

Recibido: 03/11/2011

Aceptado: 03/02/2012

\section{Resumen}

El objetivo de este estudio es describir la agenda temática de una serie de cibermedios internacionales. Para ello, nos apoyamos en los planteamientos desarrollados en la teoría de la agenda-setting a lo largo de múltiples investigaciones. Así, por medio de un análisis de contenido cuantitativo, pretendemos observar qué temas son los más relevantes dentro de las principales noticias de portada de los medios analizados, en función de determinados aspectos cómo: la autoría, la localización geográfica, las fuentes de información utilizadas o el desarrollo de las informaciones en la portada. Este artículo se enmarca dentro del proyecto de investigación del Ministerio de Ciencia e Innovación: "Evolución de los cibermedios españoles en el marco de la convergencia: análisis del mensaje" (CSO2009-13713-C05-04 -subprograma SOCI-)

Palabras clave: agenda-setting, cibermedios, análisis de contenido, temas y fuentes de información

\author{
Online Media and Agenda-Setting: \\ the Configuration of the International Media Agenda
}

\begin{abstract}
The aim of this study is to describe the thematic agenda of a number of international online media. To do this, we rely on the approaches developed in the theory of agenda-setting across multiple investigations. Thus, through a quantitative content analysis, we intend to see what issues are most relevant in the breaking news of the media analyzed according to certain aspects such as: authorship, geographic location, sources of information or the development on the front page. This article is part of the research project of the Ministry of Science and Innovation: "Evolution of Spanish online media in the context of convergence: an analysis of the message" (CSO2009-13713-C05-04-subprograma SOCI-)

Keywords: Agenda-Setting, Online Media, Content Analysis, Issues, Information Sources.

\section{Referencia normalizada}

ODRIOZOLA CHENÉ, Javier (2012): "Cibermedios y agenda-setting: la configuración de la agenda mediática internacional”. Estudios sobre el mensaje periodístico. Vol. 18, núm. 1, págs.: 157-171. Madrid, Servicio de Publicaciones de la Universidad Complutense.

Sumario: 1. Introducción. 2. Marco teórico. 2.1. Teoría de la agenda setting. 2.2. Estudios sobre el análisis del mensaje en los cibermedios. 3. Hipótesis. 4. Metodología. 4.1. Características de nuestro análisis de contenido. 4.2. Unidades de análisis y tipos de muestreo. 4.3. Categorización de nuestro análisis de contenido. 4.4. Acuerdo entre codificadores. 5. Resultados. 5.1. Una mirada general a los cibermedios estudiados. 5.2. la utilización de las fuentes de información. 5.3. Tratamiento de los temas en las informaciones. 6. Conclusiones. 7. Referencias bibliográficas.
\end{abstract}

\section{Introducción}

Este artículo se enmarca dentro del desarrollo y aplicación de la herramienta metodológica del análisis de contenido, que empleamos en el proyecto de investigación del Ministerio de Ciencia e Innovación: "Evolución de los cibermedios españoles en 
el marco de la convergencia: análisis del mensaje" (CSO2009-13713-C05-04 -subprograma SOCI-). Nuestro análisis de contenido se centra en un conjunto de cibermedios tanto nacionales como internacionales. A través de un muestreo de etapas múltiples, cada seis meses, aproximadamente, recogemos y analizamos las tres primeras noticias de cada medio hasta constituir, cada vez, una semana artificial como corpus del estudio. Dichas recogidas se están realizando en cuatro oleadas (octubre de 2010, junio de 2011, diciembre de 2011 y marzo de 2012).

A su vez, debemos tener en cuenta que la triangulación (BERGANZA y RuIz, 2005; WiMMER y DOMINICK, 2006) de diferentes herramientas metodológicas en nuestro proyecto, da relevancia a otra de las aplicaciones principales del análisis de contenido: colaborar en operaciones técnicas de una investigación (BERELSON, 1952; KRIPPENDORFF, 1990; NEUENDORF, 2002). Nuestra herramienta traza un esquema de las principales noticias de portada de los cibermedios analizados para una posterior profundización a través de otros tipos de análisis: narratológico, pragmático, retórico, hipertextual, de los elementos paratextuales, de los géneros periodísticos, de la interactividad y de la multimedialidad (ODRIOZOLA y LóPEZ, 2011: 268). Nuestro proyecto, además, se completa con un estudio etnográfico sobre las rutinas periodísticas y los cambios que se producen en las redacciones de los cibermedios analizados. Gracias a esta triangulación, podremos avanzar en el objetivo principal del proyecto ${ }^{1}$ que no es otro que "explicar cómo se construye el mensaje ciberperiodístico en España en tiempos de convergencia" (DíAZ-NOCI, SEIXA, LÓPEZ y otros, 2009: 648); entendiéndola cómo un proceso "de adaptación mutua entre viejos y nuevos medios" (MASIP, DíAZ-Noci, DoMINGO y otros, 2010: 573).

En el presente artículo analizamos los resultados obtenidos en lo concerniente a los medios internacionales en la primera oleada de estudio realizada entre el 4 y el 28 de octubre de 2010. Así, entre otros aspectos, podremos realizar un mapa temático de los cibermedios analizados con el objetivo de, posteriormente, realizar una comparación con los resultados obtenidos entre los medios nacionales. Por tanto, nos centramos en conocer los temas tratados, con el fin de conocer la agenda mediática que se desarrollan en los mismos.

\section{Marco teórico}

\subsection{Teoría de la agenda-setting}

Pese a que nuestra investigación se fundamenta en su carácter descriptivo y analítico, no podemos dejar de encuadrarla dentro de un marco teórico. Cuando hablamos de mapa temático, nos estamos refiriendo a la agenda mediática de los cibermedios analizados. Esta definición entronca con la teoría de la agenda-setting (McComBs, 2006; ShoemaKer y ReESE, 1996) cuyo origen se encuentra en el estudio de Chapel Hill (McCombs y SHAw, 1972). En un principio, los estudios relacionados con esta teoría se centran en constatar cómo el grado de énfasis puesto en los temas por las informaciones influye en la prioridad que les concede a dichos temas el público. Sin embargo,

1 Para más información sobre el proyecto se puede acceder a la página web: http://www.upf . edu/depeca/GRP/cibermedios/ 
el amplio desarrollo de esta teoría en diferentes investigaciones ha llevado a la expansión de la misma a diferentes fases del proceso comunicativo: (a) la influencia de la agenda de los medios informativos en la agenda del público; (b) el papel de la necesidad de orientación y otras condiciones contingentes a la hora de realzar o restringir su influencia; (c) la influencia de agendas externas sobre cada uno de los medios informativos; y (d) las consecuencias de toda esta actividad en las actitudes, opiniones y comportamientos (McComBs, 2006: 271-272).

De esta manera, la agenda mediática pasa a convertirse en la variable dependiente, el resultado que debe ser explicado, frente a los primeros pasos de la teoría donde ésta era entendida como un factor clave en la configuración de la opinión pública. Así como, sólo un puñado de temas son capaces de atraer la atención del público; algunos estudios también han demostrado que la agenda mediática también tiene un espacio limitado, donde los principales temas compiten por hacerse con la atención de los medios (Zhu, 1992: 834). Aquí, quedan incluidos los sitios web, que verán cómo "el alcance y el tiempo disponible de la atención pública imponen severas restricciones" (McComBs, 2006: 85). También, hay que destacar cómo, ante la pregunta de si se puede extrapolar los efectos de la agenda-setting a los cibermedios, diversos estudios han demostrado la pertinente aplicación de esta teoría a los nuevos medios surgidos en Internet (Althaus y TeWKSBury, 2002; WANG, 2000; RoBerTs, WANTA y Dzwo, 2000).

A la hora de determinar cómo se constituye la agenda mediática debemos constatar los diferentes aspectos que influyen en su constitución. SHOEMAKER y REESE identifican cinco capas de influencia: (a) la propia psicología del periodista, (b) las rutinas periodísticas, (c) las pautas organizacionales de los medios, (d) las fuentes externas (incluidos otros medios de comunicación) y (e) la ideología social predominante (SHOEMAKER y REESE, 1996: 63-250). Históricamente, la investigación de las fuentes de influencia que moldean la agenda mediática; es decir, sus orígenes, inauguró la cuarta fase de la teoría de la agenda-setting. Nuestro análisis de contenido, también, se centra en medir la relevancia de las diferentes fuentes de información utilizadas en las informaciones tratadas. Debemos tener en cuenta que, tal y como demuestran diferentes estudios, las fuentes institucionalizadas de la agenda política subvencionan los esfuerzos de las organizaciones informativas para cubrir las noticias suministrando sustanciales cantidades de información organizada, frecuentemente bajo la forma de notas de prensa redactadas en el estilo exacto de la noticias (GANDY, 1982; MANHEIM, 1994). Así, en los últimos años, se ha producido afianzamiento de los gabinetes de comunicación de los organismos oficiales, que han pasado a convertirse "en los nuevos gatekeeper de la comunicación" (PÉREZ, 2008; 254). Dicha agenda también será el objetivo de una gran variedad de grupos de presión organizados. Además, los medios de elite también influyen en la agenda mediática del resto de medios de comunicación; al igual que, el trabajo del resto de colegas periodistas, sirve como guía para validar su sentido de la relevancia informativa. Sin embargo, a la hora de tratar con las fuentes, los periodistas deben tener en cuenta que éstas no son simplemente abastecedoras de información "en tanto que obedecen casi siempre a una estrategia retórica y discursiva muy precisa, las fuentes no sólo ofrecen información, sino que además, 
con mucha frecuencia, esperan obtener un beneficio de su acercamiento a los periodistas" (MAYORAL, 2005: 94).

\subsection{Estudios sobre el análisis del mensaje en los cibermedios}

A la hora de apoyarnos en investigaciones realizadas en el ámbito de los cibermedios, tres son los estudios que marcan y guían nuestro trabajo. En primer lugar, nos servimos de labor desarrollada por GREER y MENSING, en un análisis de contenido longitudinal durante siete años de la portada de 83 diarios digitales (GrEer y MENSING, 2006). Las autoras se centran en el análisis de diferentes elementos de las noticias de portada: diseño y contenido, elementos multimedia, interactividad y posibles fuentes de ingresos. En lo que concierne al contenido, la presencia/ausencia de noticias locales y nacionales, el desarrollo del cuerpo del titular y el desarrollo del lead son las principales variables (GREER y MENSING, 2006: 20). En segundo lugar, nos referimos al trabajo desarrollado por QUANDT (2008). En él, realiza un análisis de contenido de las principales noticias de portada de diversos diarios digitales de Europa y EE.UU durante dos meses consecutivos. El estudio se centra en: (a) analizar la dependencia de las agencias de información, (b) constatar cómo se aprovechan los elementos de interactividad y multimedia y (c) identificar los temas principales, los actores y la localización geográfica. Por último, también nos servimos de la labor llevada a cabo por estudios como el coordinado por Van der Wuff. Se trata de un análisis de contenido comparativo de 51 diarios digitales e impresos europeos en un solo día (VAN DER WufF, LAUF y BALČYTIENĖ, 2008). Se centra en tres aspectos: (a) cuestiones referidas a los módulos de información presentes en la portada de los diarios (publicidad, noticias, servicios, juegos, autopromoción...), (b) cuestiones centradas en las noticias de la portada (temas, localización geográfica, tipo de ilustraciones, géneros y tipos de autoría) y (c) cuestiones centradas en las características de Internet: interacción entre usuarios, interacción usuario-productor, selección de contenido, uso de enlaces, uso de elementos multimedia y posibilidad de noticias en tiempo real (VAN DER WUFF, LAUF y BALČYTIENĖ, 2008. 410-411).

Así, el análisis de contenido se muestra como una herramienta metodológica capaz de mostrarnos una cartografía de los temas y fuentes de información de las principales informaciones. Optamos por un análisis longitudinal ya que "permite una observación de los cambios, tanto significativos como referidos a la frecuencia, a lo largo del tiempo" (GREer y Mensing, 2006: 30), por lo que resultará más apropiado para analizar los aspectos relacionados con la convergencia. En cuanto al contenido de las portadas, nuestro estudio se centra, únicamente, en los módulos de información referidos a las noticias, obviando el análisis de otros aspectos como la publicidad, los enlaces o la información de servicios. Nos centramos en las tres noticias principales de las portadas de los cibermedios analizados.

\section{Hipótesis}

Debido al carácter descriptivo de este estudio, nuestra hipótesis se centra en analizar la agenda mediática de los cibermedios analizados, en trazar un mapa temático de las principales noticias. Este mapa será estudiado en su relación con diversos factores 
como: (a) las fuentes de información; (b) la relevancia de los temas en la portada; (c) la autoría de las informaciones; y (d) la localización geográfica de los temas tratados. A su vez, aprovechándonos de nuestras unidades de registro contextuales trazaremos una pequeña descripción de cómo estructuran los diferentes cibermedios sus principales informaciones tanto en la portada como en sus nodo de desarrollo.

\section{Metodología}

\subsection{Características de nuestro análisis de contenido}

Por las necesidades de nuestra investigación nos aproximaremos al análisis desde una perspectiva descriptiva (NEUENDORF, 2002: 55). Dentro de la tipología actual del análisis de contenido nos ubicamos dentro del análisis de contenido temático (WeBER, 1990; RIFFE, LACY y FICO, 2005), que se presta "muy bien para una aplicación 'masiva' o 'extensiva'; es decir, a muestras grandes y a procedimientos estadísticos o de análisis de contenido cuantitativo" (ANDREU, 2001: web). Por otra parte, ateniéndonos a diferentes aspectos en la selección de las comunicaciones y los parámetros de medición, nuestro trabajo de investigación puede definirse como: verificativo/explicativo y frecuencial (PIÑUEL, 2002: 8-15). Verificativo y/o explicativo, porque permite dar cuenta de inferencias sobre el origen y la naturaleza de los productos comunicativos. Y frecuencial, al estudiar las posibles relaciones entre las diferentes variables.

\subsection{Unidades de análisis y tipo de muestreo}

Nuestro trabajo se centra en el análisis de diez cibermedios internacionales: Asahi Simbun. English Web Edition, guardian.co.uk, BBC Online, Le Monde.fr, Rue89, la Repubblica.it, publico.pt, Clarín.com, globo.com y NYTimes.com. Nos ocupamos de analizar las tres primeras noticias de la portada de cada medio, así como su tratamiento en el nodo de desarrollo.

Optaremos por un muestreo multi-etápico (KRIPPENDORFF, 1990: 99-100). Para ello, a lo largo de un mes construiremos una semana artificial de análisis. Podemos constatar cómo "un tamaño de muestra de seis días será eficaz y efectivo para representar el contenido de los sitios de noticias actualizados en un periodo de seis meses" (WANG y RiFFe, 2006: web).

\subsection{Categorización de nuestro análisis de contenido}

Contaremos con una serie de unidades contextuales: (a) Cibermedio; (b) Fecha; (c) Posición en la portada; (d) Distribución espacial; (e) Uso de recursos audiovisuales en la portada; (f) Relevancia en la portada; (g) Uso de imágenes; (h) Uso de gráficos; (i) Uso de audio; (j) Uso de video; (k) Autor; (l) Localización; y (m) Posibilidad de introducir comentarios. Estas unidades nos permitirán trazar una rápida descripción de los cibermedios, además de permitirnos profundizar en el estudio de nuestras variables principales.

Las fuentes de información serán una de estas dos variables. Analizaremos: (a) Número de fuentes utilizadas; (b) Identidad de la primera fuente del titular; (c) Identidad de la primera fuente del texto; (d) Función de la primera fuente del texto; (e) Identidad de la segunda fuente; (f) Función de la segunda fuente. 
En cuanto a los temas de las informaciones nos hemos centrado en identificar el principal de cada información, con el fin de evitar posibles desacuerdos que condicionen la fiabilidad de nuestro análisis. Para identificar los temas, partimos de los códigos propuestos por el International Press and Telecommunications Council para la transferencia internacional de noticias (Del VAlle y García, 2002: 115). A estas categorías, los pre-test realizados, nos han impulsado a introducir tres nuevas categorías: (q) Tráfico; (r) Sucesos; y (s) Otros.

\subsection{Acuerdo entre codificadores}

La fiabilidad entre codificadores (LOMBARD, SNYDER-Duch y CAMPANELLA, 2002) de nuestro análisis de contenido se sitúa en un 0,87 (ODRIOzOLA y LÓPEZ, 2011: 264265). Dicha fiabilidad la hemos obtenido, mediante la combinación de métodos liberales y conservadores.

\section{Resultados}

\subsection{Una mirada general a los cibermedios analizados}

Si bien, el objetivo de nuestra investigación es realizar un mapa temático y de fuentes utilizadas por los diferentes medios analizados, no podemos dejar de hablar sobre algunos aspectos de los mismos que se encuentran presentes entre nuestras variables de estudio. Por tanto, a continuación, nos centraremos en analizar algunas cuestiones como: (a) la distribución espacial; (b) la utilización de recursos audiovisuales; (c) la relevancia de las informaciones en la portada; (d) el uso de comentarios; (e) la autoría; y (f) la localización.

A la hora de encajar las informaciones en sus portadas, los cibermedios analizados optan por enmarcarlas, principalmente, en las primera columna (49\%), además de en la segunda (30\%). Sin embargo, podemos constatar cómo aquellas informaciones más destacas pueden ocupar el espacio formado por la suma de las dos columnas $(14,8 \%)$. Frente a ellas, también nos encontraremos con aquellas informaciones donde la columna se divide en dos, limitando la visibilidad de las mismas $(5,7 \%)$. Sólo una información, ha sido capaz de utilizar las tres columnas teóricas, en las que dividimos la portada de los cibermedios.

En cuanto a la utilización de recursos audiovisuales, podemos constatar cómo más de la mitad de las informaciones analizadas cuentan con recursos audiovisuales en su tratamiento de portada, 59,5\%. Si nos fijamos en la utilización de estos recursos, tanto en la portada como en el primer nodo de desarrollo de las informaciones, observamos cómo la fotografía es el recurso audiovisual más utilizado. El 86,2\% de las informaciones incorporan un apoyo fotográfico. Esta alta presencia se contrapone con la del resto de recursos: gráficos $(13,8 \%)$, audio $(4,8 \%)$ y video $(19 \%)$.

A través de una serie de baremos hemos medido la relevancia de las informaciones analizadas en las portadas en las que se ubican. Dicho baremo se ha construido partiendo de: (a) la posición en la portada, (b) el número de columnas que ocupan las informaciones y (c) los recursos audiovisuales en la portada. Hemos introducido, a su vez, una serie de valores que corrigen nuestras mediciones. Se trata de otorgar un punto negativo (a) cuando las informaciones solo cuentan con un título sin ningún 
desarrollo y (b) cuando las informaciones ocupan menos de una columna. Así, la relevancia en la portada de las informaciones se situará entre unos valores que van desde el -2 (tercera noticia, sin recursos audiovisuales, ocupa menos de una columna y sólo es un título) hasta 6 ( $1^{\mathrm{a}}$ noticia, ocupa las tres columnas, posee más de dos recursos audiovisuales). Constatamos como la puntuación media de las informaciones analizadas es de 1,9. Más adelante nos valdremos de esta variable para constatar aspectos como los temas más relevantes en las portadas de los cibermedios.

En un $49,5 \%$ de las informaciones, los usuarios de los cibermedios tienen la posibilidad de incorporar comentarios a las informaciones. Sin embargo, la situación varía dependiendo del medio analizado. Así, Le Monde.fr, Rue89 y publico.pt ofrecen la posibilidad de introducir comentarios en todas sus informaciones. Por el contario, en el Asahi Simbun. English Web Edition, nunca se ofrece esta posibilidad.

Debemos constatar como la mayor parte de las informaciones analizadas van firmadas por periodistas propios de los medios analizados, $68,1 \%$. Dentro de estas categoría, será la figura del redactor la que firme la mayor parte, $67,8 \%$; seguido de la firma genérica, $18,2 \%$ y del corresponsal, $11,9 \%$. Otras figuras como la del enviado especial no cuentan con apenas espacio, $1,4 \%$. Tras las informaciones propias se encuentran aquellas informaciones que carecen de ningún tipo de firma, 22,4\%. Las informaciones de autoría mixta suponen un 7,1\% del total de la muestra. En este caso, lo que priman son las informaciones construidas a partir de datos propios y de los proporcionados por agencias, aunque, en una ocasión, encontramos una información redactada entre los redactores del cibermedio y los propios usuarios, y otra información firmada en autoría compartida con otro cibermedio. Por último, el 2,4\% de las informaciones analizadas provienen directamente de agencias.

Si nos atenemos a los datos, podemos comprobar cómo las informaciones del entorno son las que dominan frente a las de carácter mundial. Así, el carácter nacional domina en un $47,1 \%$ de las informaciones. A este carácter próximo ayuda, también el que el $14,8 \%$ de las informaciones tengan un carácter regional o local. Por otro lado se encontrarían aquellas informaciones cuya relevancia es internacional, 26,7\%, o aquellas donde la relevancia no llega a ser mundial pero que afectan a una serie de pa-

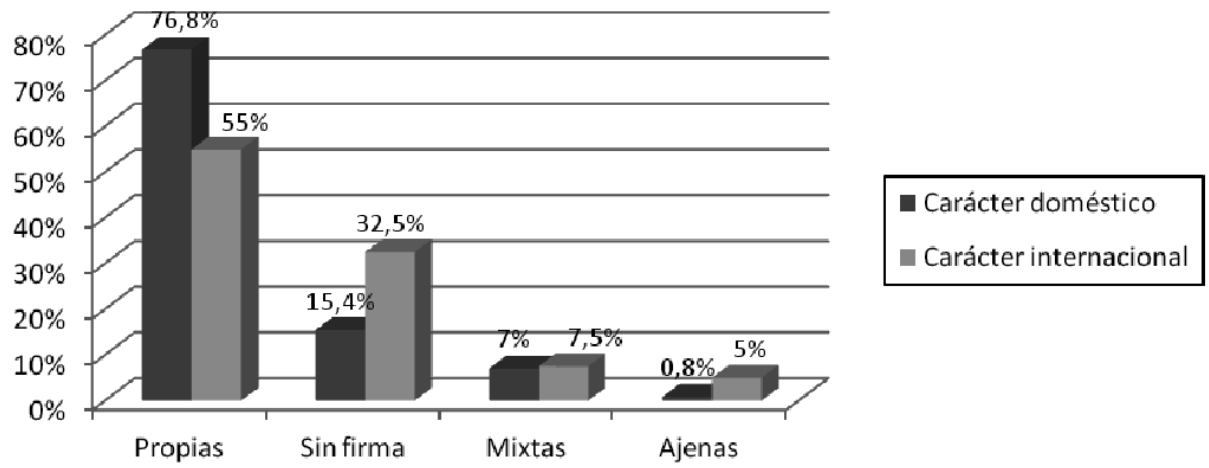

Figura 1 Comparativa de la autoría de las informaciones en función de su localización. Fuente: Elaboración propia 
íses que comparten un entorno geográfico. Así, las informaciones de carácter continental suponen el $11,4 \%$. Atendiendo a si los temas tratados son de carácter doméstico (Local/regional o Nacional) o, por el contrario, poseen una visión más internacional (Continental o Internacional) podemos constatar como las noticias de autoría propia aumentan considerablemente, $76,8 \%$, en los asuntos domésticos frente a los temas internacionales, $55 \%$. Ese hueco es ocupado por un aumento de las informaciones sin firma, que pasan del 15,4\% al 32,5\%, y de las ajenas, principalmente de agencias, que pasan de un $0,8 \%$ al $5 \%$.

\subsection{La utilización de las fuentes de información}

Comenzaremos diciendo que la media de fuentes consultadas en cada información es de 3,3. Con un uso de fuentes superior a la media del análisis nos encontramos cibermedios como: (a) guardian.co.uk, 5,3; (b) NYTimes.com, 4,9; (c) Rue89, 4,4; o Asahi Simbun. English Web Edition, 4,2. En el lado opuesto se encontrarían: (a) Clarín.com, 1,4; (b) publico.pt, 1,6; y (c) globo.com, 2. Otro factor que influye en el número de fuentes utilizada es el que hace referencia a la autoría de las informaciones. Viendo los resultados de nuestro análisis, podemos constatar cómo las informaciones que cuentan con un mayor número de fuentes, por término medio, son las propias, 3,6 ; y las mixtas, 3,5. Las informaciones no firmadas cuentan con una media de fuentes de 2,7; mientras que la media de fuentes utilizadas en las informaciones ajenas se sitúa en 1,4.

Debemos destacar como, por normal general, los cibermedios analizados no destacan ninguna fuente en la mayoría de sus titulares. El 69,1\% de las informaciones no incorporan ninguna fuente en sus títulos, antetítulos o subtítulos.

En nuestro análisis hemos estudiado, únicamente, las dos primeras fuentes de cada texto informativo. Pese a ello, comprobamos cómo en estas dos primeras fuentes se encuentran las principales o únicas de la información. Así, excluyendo las informaciones que no recurren a ninguna fuente, $8,1 \%$; podemos observar como la fuente principal (incluyendo, evidentemente, aquellas donde solo hay una) se encuentra entre las dos primeras en el 88,6\% de los casos. Podemos observar cómo entre las fuentes utilizadas en los medios analizados, algunas de ellas son más proclives a convertirse en la iniciadora del texto (fuente principal o fuente única); mientras que otras aumentan su presencia cuando ayudan a complementar o confrontar la información de otras fuentes (fuentes secundarias). Así, las categorías "Gobierno y oposición parlamentaria"; "Otros miembros de las AA.PP"; "Expertos/científicos" presentan unos valores superiores cuando son utilizadas como fuente principal o única; mientras que otras como: "Grupos de presión", "Fuente sin relevancia social" y "Otras fuentes de relevancia social", aumentarán su presencia cuando tengan una función secundaria. Esta función complementaria está muy reforzada en el caso de las fuentes sin relevancia social. La categoría "Otros medios de comunicación" presenta unos números similares en ambos casos. Lo más relevante de este tipo de fuentes, es su alta presencia como fuente única, $25 \%$, muy superior a los valores del resto de fuentes. Las tres categorías restantes: "Grupos terroristas/criminales"; "Fuentes anónimas"; y "Otros", cuentan con una única mención a lo largo del análisis, por lo que resulta contraproducente establecer conclusiones. 


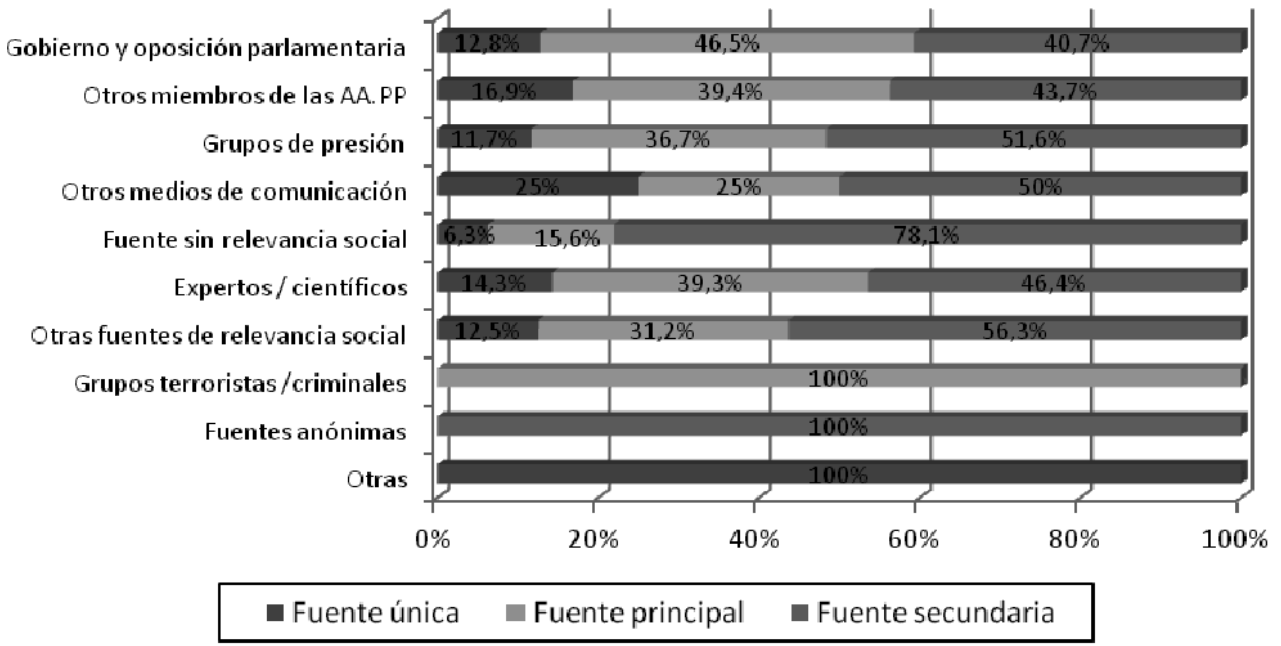

Figura 2 Función de las fuentes de información. Fuente: Elaboración propia

\subsection{Tratamiento de los temas en las informaciones}

La política es el tema más tratado, con diferencia en el conjunto del análisis, 35,7\%. Las guerras y conflictos son los segunda cuestión más tratada en los cibermedios analizados, $13,8 \%$, aunque su presencia es muy inferior a la de los temas políticos. Estos últimos, serán los únicos presentes en todos los cibermedios analizados. Los temas relacionados con la economía, las finanzas y los negocios son el tema del 9,1\% de las informaciones analizadas. Si bien, su presencia es inferior a la de los temas relacionados con la guerra y otros conflictos, es más constante en cuanto a número de cibermedios en los que aparece.

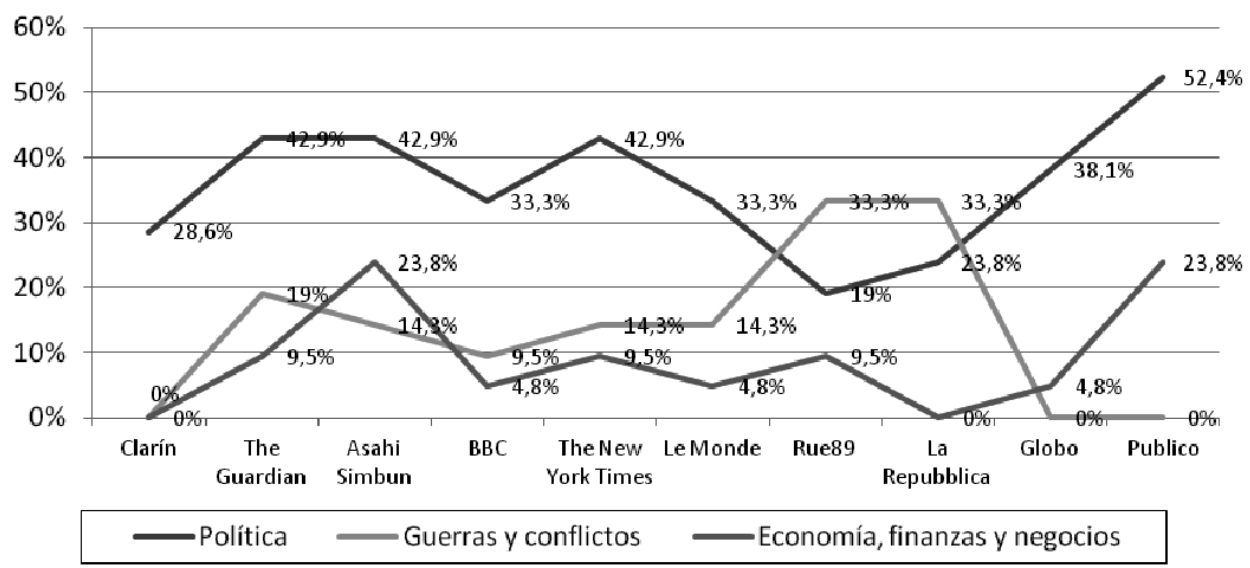

Figura 3 Presencia de los temas más tratados en cada uno de los cibermedios. Fuente: Elaboración propia 
Tras estos, se sitúan los temas relacionados con desastres y accidentes, 7,1\%. Que se apoyan principalmente, en su elevada presencia en la $B B C, 23,8 \%$ y Globo, $14,3 \%$. Algo parecido sucede con los sucesos, que cuentan con una presencia media en el análisis del 6,2\% sustentada en su alta presencia en dos cibermedios: Clarín, 23,8\%, y La Repubblica, 23,8\%. En contraposición a esta distribución temática se encuentran los asuntos judiciales. Su presencia es limitada, 5,7\%, pero más constante en el conjunto de cibermedios analizados. La presencia de los deportes, 5,2\%, también se sustenta en sólo cuatro medios que cuentan con estos temas en su portada. Por su parte, los temas relacionados con el mundo de la cultura, del arte y de los espectáculos centran el 4,3\% de las informaciones analizadas.

Hay otros temas que cuentan con espacio en las informaciones principales. Sin embargo, su presencia es tan escasa, que preferimos esperar a ver su evolución en las próximas oleadas de estudio.

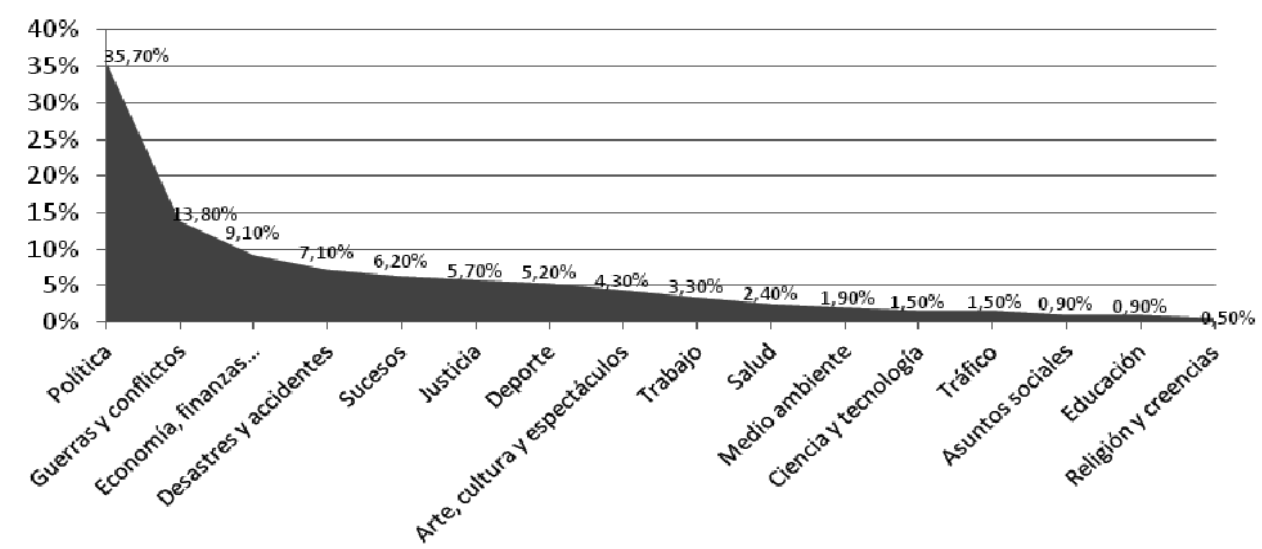

Figura 4 Presencia de los temas en el total del análisis. Fuente: Elaboración propia

A parte de esta visión panorámica de los temas tratados en los cibermedios, nos apoyamos en otras variables de nuestro análisis como: (a) la relevancia, (b) la autoría o (c) la localización, con el objetivo de profundiza en nuestro análisis. Así, tomando las informaciones que cuentan con una puntuación, en cuanto a la relevancia en la portada, superior a 2. Podemos observar como la política sigue dominando, al ser el tema principal en el 33,3\% de las informaciones. Sin embargo el ascenso más significativo se produce en la categoría "Guerras y conflictos" cuya presencia se eleva hasta un $25 \%$ (casi el doble que en el conjunto de todas las informaciones). Por su parte, los temas económicos ven reducida su presencia entre los temas más relevantes, $6,6 \%$; viéndose superada por cuestiones como los desastres y accidentes, $11,7 \%$, y los sucesos, 11,7\%. Entre el resto de temas, sólo los asuntos relacionados con la salud presentan unos valores superiores a su media en el conjunto del análisis. También estudiamos si la autoría influye en los temas tratados. Los tres temas principales aumentan su presencia en las informaciones de autoría propia, en especial los temas económicos. Como consecuencia, los siguientes temas ven limitado su espacio, disminuyendo, sobre todo, los temas relacionados con: (a) desastres y accidentes; y (b) 
deporte. Por otro lado, podemos observar cómo la política domina tanto en las informaciones de carácter nacional, incluyendo las de ámbito local/regional, como en las de carácter internacional, incluyendo las de ámbito continental. Sin embargo, algunos de los temas tratados sí que tenderán a circunscribirse a un determinado ámbito. El caso más claro es el de los sucesos que no cuentan con ninguna mención en el ámbito internacional. Los deportes, al igual que la economía o la justicia, también aumentarán su presencia en cuestiones de ámbito nacional o regional. En el lado contrario, se encuentran los desastres y accidentes, que crecen de manera considerable en el ámbito internacional. También tienden a tener más presencia en este ámbito asuntos como las guerras y conflictos o la salud.

Por último, también consideramos necesarios profundizar en las relaciones de los temas con sus fuentes de información, centrándonos en tres aspectos: (a) el número de fuentes utilizadas, (b) los tipos de fuentes utilizadas en los temas y (c) las fuentes principales de los temas principales. Entre los temas principales, la categoría "Guerras y conflictos" es el que incorpora un mayor número de fuentes en sus informaciones. De hecho, entre los cinco temas principales (política, guerras y conflictos, economía, desastres y accidentes, y sucesos), que suponen el $81,4 \%$ de los temas tratados en los cibermedios, sólo los acontecimientos relacionados con guerras y conflictos presentan unos números superiores a la media del análisis, 5,1. También presentan valores superiores a la media otros temas como: arte, cultura y espectáculos", 5,1; o trabajo, 4,1. Sin embargo, serán los asuntos sociales los que utilicen un mayor número de fuentes en sus textos, 7. A pesar de ello, debemos tener en cuenta, que este tema sólo es el principal en dos informaciones del total del análisis, por lo que esperaremos a próximas oleadas para constatar su evolución. Los temas que menos fuentes, por término medio, presentan en sus informaciones son religión y creencias, 0 ; tráfico, 1 ; y deportes, 1,4. En los dos primeros casos, su baja presencia en el análisis, $0,5 \%$ y $1,5 \%$, respectivamente, aconseja, esperar a próximas oleadas para extraer posibles conclusiones. En el caso de los deportes, la presencia de crónicas en directo de diferentes acontecimientos deportivos, donde el propio periodista se convierte en testigo de la información, puede limitar el uso de fuentes de información.

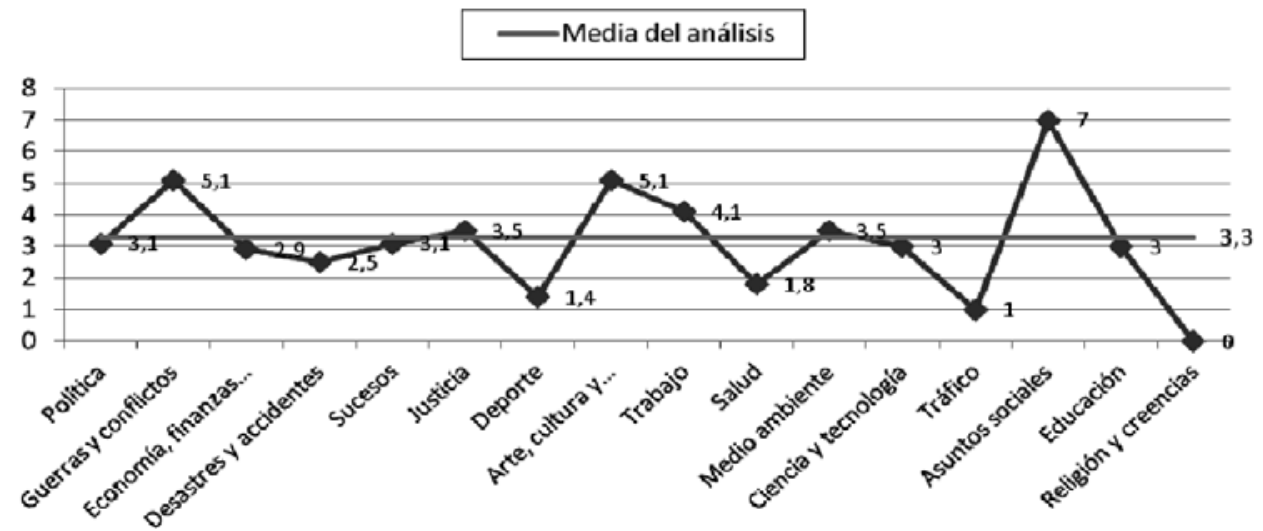

Figura 5: Número de fuentes utilizadas en función del tema principal. Fuente: Elaboración propia 
La distribución de las fuentes en los diferentes temas tratados en las informaciones, nos muestra como la categoría "Gobierno y oposición parlamentaria" domina en: política, economía y asuntos Sociales. Por su parte, la categoría referida a "Otros miembros de las AA.PP" será la más utilizada en las categorías: guerras y conflictos, sucesos, justicia, trabajo, tráfico y educación. Los "Grupos de presión" cuentan con una considerable presencia en los temas principales, pero sólo dominarán en las cuestiones relacionadas con salud y educación. Cuando los cibermedios se ocupen de asuntos relacionados con desastres y accidentes, se servirán de otros medios de comunicación como fuente más utilizada. También dominará en los temas relacionados con la justicia, junto con la categoría "Otros miembros de la AA.PP". La categoría "Expertos" domina en las cuestiones relacionadas con ciencia y tecnología y con educación; junto con la categoría "Fuentes sin relevancia social". La categoría "Otras fuentes de relevancia social" dominan en las cuestiones relacionadas con arte, cultura y espectáculos. También crece su presencia en los deportes. El resto de categorías tienen una presencia tan escasa que no consideramos oportuno establecer conclusiones. Por último, debemos mencionar como en algunos temas como: justicia, deportes, tráfico y religión y creencias, lo más habitual es que no se utilice ninguna fuente de información.

Al centrarnos en las fuentes principales de los principales temas tratados (política; guerras y conflictos; y economía), podemos comprobar cómo la categoría "Gobierno y oposición parlamentaria" domina, claramente, los temas políticos, limitando el espacio del resto de categorías. Sin embargo, al hablar de guerras y conflictos, serán los grupos de presión quienes logren acceder a ese primer puesto como fuente principal, seguida de la categoría "Otros miembros de las AA.PP". Al hablar de asuntos económicos, "Gobierno y oposición parlamentaria" vuelven a recuperar el primer puesto, apoyando su dominio en la categoría "Otros miembros de la AA.PP". Por otro lado, categorías como "Expertos" y "Otros medios de comunicación", mantienen una presencia constante en los tres temas principales. En último lugar, las categorías "Grupos terroristas" y "Fuentes sin relevancia social" consiguen acceder de forma puntual a algunos de estos temas como fuentes de información principal.

\section{Conclusiones}

Los cibermedios internacionales analizados tienden a introducir sus principales noticias en sus dos primeras columnas. En algunas ocasiones, algunas informaciones consiguen unir estas dos columnas aumentando su relevancia. Las informaciones de portada suelen ir acompañadas de una fotografía, aumentando su presencia en los nodos de desarrollo. El resto de recursos audiovisuales (audio, video, información gráfica) ven muy limitado su espacio. Así, el esquema básico de una información de portada será la que ocupa una columna y va acompañada de una fotografía. Si nos centramos en el nodo de desarrollo de las informaciones, observamos cómo en una de cada dos informaciones se da la posibilidad de introducir comentarios por parte de los usuarios. En cuanto a su localización dominan, ligeramente, los temas de carácter doméstico frente a los internacionales. Esto afecta a la autoría de las mismas, cre- 
ciendo el dominio las informaciones propias en las cuestiones estatales. En el ámbito internacional, el dominio es menor, ocupando ese espacio los textos sin firma y los provenientes de agencias.

Pero, el objetivo de este estudio era comprobar la agenda mediática con el fin de trazar un mapa de contenidos de los cibermedios analizados. Así, la categoría "Política" domina claramente en función de diferentes criterios: (a) frecuencia, (b) relevancia en la portada, (c) frecuencia en informaciones propias, (d) localización geográfica. Otros dos temas que cuentan con un espacio destacado y continuo en el conjunto del análisis son: "Guerras y conflictos" y "Economía, finanzas, negocios...". El primero de ellos se caracterizará por: (a) aumentar considerablemente su frecuencia cuando nos ocupamos de los temas con una mayor relevancia en la portada; (b) crecer su presencia en las informaciones de autoría propia; y (c) tener una mayor presencia en cuestiones internacionales frente a las domésticas. Por su parte, la presencia de los temas económicos: (a) disminuye, entre los temas con una mayor relevancia en la portada; (b) aumenta, entre los temas redactados por los profesionales de los propios cibermedios; $y$ (c) es mayor, en las cuestiones domésticas frente a los asuntos de carácter internacional.

Por último, debemos ser conscientes que uno de los factores que más influyen en la configuración de la agenda mediática de los medios son las fuentes de información. Observamos algunas cuestiones interesantes. Así, los temas más tratados, a excepción de la categoría "Guerras y conflictos", suelen contar con un número de fuentes en sus informaciones inferior a la media del análisis, lo que realza el valor de las fuentes que acceden a esos textos. El dominio de las fuentes institucionales se concreta al observar cómo dos categorías dominan la mayoría de los temas: (a) "Gobierno y oposición parlamentaria" (política, economía y asuntos sociales), y (b) "Otros miembros de las AA.PP”. (guerras y conflictos, sucesos, justicia, trabajo, tráfico y educación). Junto a ellas, sólo la categoría "Expertos" es más proclive a iniciar o fundamentar los textos periodísticos, como fuente principal o única e las informaciones.

\section{Referencias bibliográficas}

ALTHAUS, Scott L. and TEWKSBURY, David (2002): "Agenda Setting and the 'New' News. Patterns of Issue Importance Among Readers of the Paper and Online Versions of the New York Times". Communication Research, vol. 29, $\mathrm{n}^{\mathrm{o}} 2$. Thousand Oaks, CA, Sage, pp. 180-207.

ANDREU, Jaime (2001): "Las técnicas de Análisis de Contenido: Una revisión actualizada", en Centro de Estudios Andaluces: http://public.centrodeestudiosandaluces.es/pdfs/S200103.pdf. [fecha de consulta: 10 de enero de 2010]

BERELSON, Bruce (1952): Content Analysis in communication research. New York, Hafner.

BERGANZA, María Rosa y RUIZ, José Antonio (2005): Investigar en comunicación. Guía práctica de métodos y técnicas de investigación social en comunicación. Madrid, McGraw-Hill. 
DEL VALLE, Félix y GARCÍA, Antonio (2002): “Construcción de un tesauro para el Centro de Documentación de Telecinco". Scire, no 8 (1). Zaragoza, Universidad de Zaragoza, pp.103-118.

DÍAZ-NOCI, Javier; SEIXAS, Lia; LÓPEZ, Guillermo y otros (2009): "Content and message analysis of online journalism. Some methodological proposals". Trípodos (extra 2009). V Congrés Internacional. Comunicació y realitat. Barcelona, Facultad de Comunicación Blanquerna, pp. 647-656.

GANDY, Óscar (1982): Beyond the Agenda-Setting. Information Subsidies and Public Policy. New Jersey, Ablex.

GREER, Jeniffer and MENSING, Donica (2006): "The Evolution of Online Newspapers. A Longitudinal content analysis, 1997-2003", en XIGEN, Li: Internet newspapers. The making of a mainstream medium. Mahwah, NJ, Lawrence Erlbaum, pp.13-33.

KRIPPENDORFF, Klaus (1990): Metodología del análisis de contenido. Teoría y práctica. Barcelona, Paidós.

LOMBARD, Matthew, SNYDER-DUCH, Jennifer y CAMPANELLA, Cheryl (2002): "Content Analysis in Mass Communication. Assessment and Reporting of Intercoder Reliability". Human Communication Research, vol. 28, n 4 . New Jersey, International Communication Association, pp. 587-604.

MANHEIM, Jarol (1994): Strategic Public Diplomacy and American Foreign Policy. The Evolution of Influence. Nueva York, Oxford University Press.

MASIP, Pere; DÍAZ-NOCI, Javier; DOMINGO, David y otros (2010): "Investigación internacional sobre ciberperiodismo: hipertexto, interactividad, multimedia y convergencia”. El profesional de la información, vol. 19, n 6 . Valencia, Universidad Politécnica de Valencia, pp. 568-576.

MAYORAL, Javier (2005): "Fuentes de información y credibilidad periodística". Estudios sobre el Mensaje Periodístico, vol. 11. Madrid, Servicio de Publicaciones Universidad Complutense de Madrid, pp. 93-102.

McCOMBS, Maxwell (2006): Estableciendo la agenda. El impacto de los medios en la opinión pública y en el conocimiento. Barcelona, Paidós.

McCOMBS, Maxwell (2005): "A Look at Agenda-Setting. Past, Present and Future". Journalism Studies, vol. 6. Oxford, Routledge, pp. 543-557.

McCOMBS, Maxwell y SHAW, Donald (1972): "The agenda-setting function of mass media". Public Opinion Quarterly, vol. 36. Oxford, Oxford University Press, pp. 176-187.

NEUENDORF, Kimberly (2002): The content analysis guidebook. Thousand Oaks, CA, Sage.

ODRIOZOLA, Javier y LÓPEZ, Guillermo (2011): “Content analysis applied to Digital Media. A Comparison of News in The Guardian, Clarin and Asahi Simbun, en SALAVERRÍA, Ramón (ed.): Diversity of Journalisms. Proceedings of the ECREA 
Journalism Studies Section and 26th International Conference of Communication (CICOM). Pamplona, Universidad de Navarra, pp. 261-270.

PÉREZ, Concha (2008): “Comunicación política. Un reto para la especialización de periodistas y fuentes". Ámbitos, $\mathrm{n}^{\circ}$ 17. Sevilla, Universidad de Sevilla, pp. 251-269.

PIÑUEL, Jose Luis (2002): "Epistemología, metodología y técnicas del análisis de contenido". Estudios de Sociolingüística, n 3 (1). Vigo, Universidad de Vigo, pp. $1-42$.

QUANDT, Torsten (2008): “(NO) News on the World Wide Web? A comparative content analysis of online news in Europe and the United States". Journalism Studies, vol. 9, issue 5. Oxford, Routledge, pp. 718-738.

RIFFE, Daniel, LACY, Stephen y FICO, Frederick (2005): Analyzing media messages: using quantitative content analysis in research. New York, Lawrence Erlbaum.

ROBERTS, Marilyn, WANTA, Wayne and DZWO Tzong-Horng (2002): "Agendasetting and issue salience online". Communication Research, vol. 29, $\mathrm{n}^{\circ} 4$. Thousand Oaks, CA, Sage, pp. 452-465.

SHOEMAKER, Pamela, y REESE, Stephen (1996): Mediating the Message. Theories of Influences on Mass Media Content. New York, Longman.

VAN DER WUFF, Richard, LAUF Edmund, BALČYTIENĖ, Auksė y otros (2008): "Online and newspaper in Europe in 2003. Evolving towards complementarity". Communications. The European journal of communication research, $\mathrm{n}^{\circ} 33$. Berlín, De Gruyter, pp. 403-430.

WANG, Tai-Li (2000): “Agenda Setting Online. An Experiment Testing The Effects of Hyperlinks in Online Newspapers". Southwestern Mass Communication Journal, vol. 15, $\mathrm{n}^{\circ}$ 2. Texas, Southwest Education Council for Journalism and Mass Communication, pp.59-70.

WANG, Xiaopeng and RIFFE, Daniel (2006): “An Exploration of Samples Sizes for Content Analysis of the New York Times Web Site" en Web Journal of Mass Communication Research, vol. 20: http://www.scripps.ohiou.edu/wjmcr/vol20/20.html [fecha de consulta: 10 de enero de 2010]

WEBER, Robert Philip (1990): Basic content analysis. Thousand Oaks, Sage.

WIMMER, Roger y DOMINICK, Joseph (2006): Mass media research. An introduction. Belmont, CA, Thomson.

ZHU, Jian-Hua (1992): "Issue competition and attention distraction. A zerosum theory of agenda setting". Journalism Quarterly, vol. 69, $\mathrm{n}^{\circ}$ 4. Iowa, American Association of Schools and Departments of Journalism, pp. 825-836. 